\title{
EMG activity of upper limb on spinal cord injury individuals during whole-body vibration
}

\author{
USLG Da Silva ${ }^{1}$, HA Villagra ${ }^{1}$, LL Oliva $^{2}$, NF $_{\text {Marconi }}{ }^{3}$ \\ ${ }^{1}$ Department of Education, Physical Activity and Human Motor Control, Autonomous University of Madrid, \\ Madrid, Spain \\ ${ }^{2}$ Department of Sciences, Physiotherapy and Rehabilitation, Universidad Rey Juan Carlos, Madrid, Spain \\ ${ }^{3}$ Physical Therapy Department, College of Health and Human Sciences, Western Carolina University, \\ Cullowhee, NC, USA
}

Received: September 14, 2015

Accepted: August 2, 2016

\begin{abstract}
Whole-body vibration (WBV) has shown positive results increasing electromyographic (EMG) activity and strength in a healthy population when applied to upper and lower limbs. The aim of this study was to verify if WBV increases the EMG signal of the upper limb muscles in patients with spinal cord injury (SCI). For this study, 15 patients with $\mathrm{SCI}$ were selected and one single session of WBV was applied to the upper limb. Five sessions of $30 \mathrm{~s}$ at $30 \mathrm{~Hz}$ were applied and EMG of anterior deltoid and forearm muscles was measured. The results show that EMG activity had a significant increase during WBV session compared with baseline muscle activity. These results support WBV as an efficient tool to increase the upper body EMG in individuals with SCI.
\end{abstract}

Keywords: whole-body vibration, spinal cord injury, electromyography, upper limb, vibratory platform

\section{Introduction}

An individual with spinal cord injury (SCI) and paralysis of the lower limbs must transfer the weight bearing demands of daily tasks to the upper extremities. A deleterious consequence of this shift in load to the upper extremities is an increased prevalence of shoulder pain with activities such as manual wheelchair propulsion. Although wheelchair propulsion contributes to shoulder pain in persons with SCI, the shoulder joint reaction forces experienced during level propulsion are low to moderate in magnitude. The repetitive nature of manual wheelchair propulsion, however, places muscles that are more intensely active at a higher risk for fatigue, which may predispose the shoulder complex to impingement and muscle and tendon injuries (17).

One of the latest neuromuscular training methods which has been quickly developing and gaining popularity is the so-called whole-body vibration (WBV) training. It has become increasingly popular with many coaches, trainers, and athletes for conditioning, rehabilitation, and general fitness (21). Recently, there has been a growing interest in the neuromuscular effects of WBV in training and rehabilitation settings (16).

The application of vibration to an active muscle has recently been shown to cause a shift in electromyography (EMG) patterns $(2-7,10,12,13)$.

\footnotetext{
Corresponding author: Uriel Sena Lopes Gomes Da Silva

Department of Education, Physical Activity and Human Motor Control, Autonomous University of Madrid Madrid, Spain

Phone: +34 608324588; E-mail: uriel_fisio@hotmail.com
} 
WBV applied over upper limbs has increased the muscular performance and EMG activity in a healthy population $(8-11,15)$.

Vibrations applied to the arm showed enhancement of mechanical power and an increase in neuromuscular efficiency supporting the evidence that vibrations represent a strong stimulus for the neuromuscular system. EMG activity during vibrations has been shown to reach values higher than $200 \%$ baseline in arms' flexor muscles $(1,2)$.

Muscle activation during vibration can be monitored by recording EMG signal to the target muscles. With this tool, it is in fact possible to determine the muscle activity in a given task. In light of the above, it is possible to affirm that EMG can be used to provide an indication of the muscle activity determined by vibration. In fact, EMG signal can be used to measure the magnitude of muscle activation following the application of vibration (2).

Few articles have studied the acute effects of WBV as a muscle training method applying a single session of vibration. The results on muscle strength and performance and EMG signal have been positive (4).

Limitations of previous studies are differentiations in the training procedures regarding exercise parameters (side alternating or synchronous vibration platform type, duration of WBV, and volume of WBV exercises) and the biomechanical variables (vibration frequency, vibration amplitude, and joint angle) determining the vibration load (18). This is why in this study, we have used the protocols that produced satisfactory results over upper limbs EMG.

A significantly higher level of EMG activity appears in muscles during WBV treatment with respect to a rest condition; specific WBV frequencies also seem to produce a higher EMG signal than others (7).

The analysis of surface EMG (sEMG) and its concise parameters is a common method of analysis of muscle activity (6).

Beneficial effects of WBV exercises are believed to result, among others, from augmented neuromuscular activity during the exposure to the vibration (13).

WBV exercises acutely increase muscle activity during the exposure, as shown in numerous studies with the help of sEMG recordings $(2,13,16,18)$.

Greater vibration frequencies $(30 \mathrm{~Hz})$ and amplitudes $(3-5 \mathrm{~mm})$ induce a higher muscle activity than the lower frequencies and/or amplitudes $(10,14,16)$.

The aim of this study was to identify the EMG activity of upper limb in individuals with SCI during a single session of WBV. We have used the parameters that received the best results on upper limb in a distinct population while trying to avoid the limitations of the previewed studies.

\section{Methods}

\section{Experimental study}

Subjects. For this study, 15 individuals with SCI ( $46 \pm 20$ years) were recruited. All subjects had their injury level beneath T3 and were all wheelchair users for more than 1 year ( $11 \pm 10$ years) prior to the intervention. To participate in this study, they should have been using a manual wheelchair more than $1 \mathrm{~h}$ per day and have no orthopedic issues concerning the upper limb. They cannot have any contraindications to WBV such as epilepsy, active tumor, or severe arthrosis either.

Prior to data collection, participants were informed of the requirements associated with participation and provided written informed consent. Participants were encouraged to 
maintain their dietary, sleeping, and drinking habits during participation in the study. This study is in line with all international ethical standards for human studies and was duly approved by ethics committees of the University involved. It also consists of approval by the ethics committee of the centers participating and authorization by all volunteers.

WBV session. WBV intervention was composed of one single session, five series of $30 \mathrm{~s}$ vibrations with $1 \mathrm{~min}$ rest between. The frequency employed was $30 \mathrm{~Hz}$, while the amplitude was kept constant at $4 \mathrm{~mm}$. The vibratory platform model Galileo Advanced Novotec Medical had been used for this study. The position assumed on the platform was to support the upper limbs over the platform with elbows and forearm (Fig. 1). Forearm had been completely supported over the platform with elbows opened underneath shoulders. Hands were kept together with the eyes looking to the horizon. Trunk and lower limb had assumed the most comfortable and possible position to each subject respecting personal limitations. This position had been chosen to make the WBV session possible and comfortable to the subjects.

$E M G$. EMG signal was taken from anterior deltoid and anterior forearm of the dominant limb. These muscles were chosen due to their agonist function during the wheelchair propulsion. The skin was shaved and cleaned with alcohol prior to sticking the electrodes. Dormo Silver Chloride bipolar surface electrodes were used in this study. Electrodes were fixed longitudinally over muscle belly, $2 \mathrm{~cm}$ distance from each other according to SENIAM indications. The reference electrode was located on the ankle of the same leg and EMG cables were secured appropriately so as to prevent the cables from swinging and from causing movement artifact. Bipolar surface electrodes were connected through the cables to the converter Biometrics E-LINK inter X Sensor Type GX4 (SX230FW EMG amplifier, 4 mm snap). This filter converts a negative value to a positive value of the same magnitude. No filter constant is required. Data had been collected using Biometrics E-LINK software.

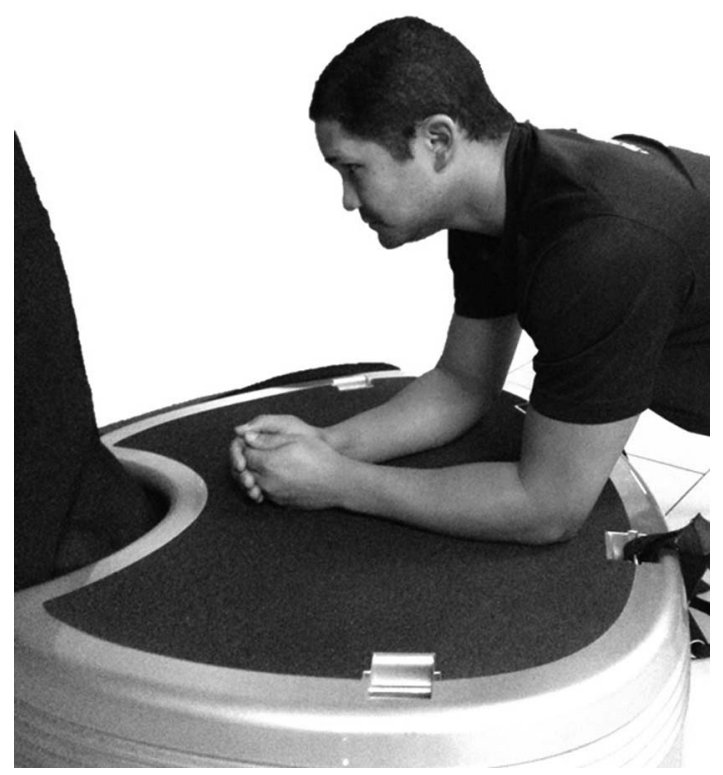

Fig. 1. Representation of the positioning over the platform 


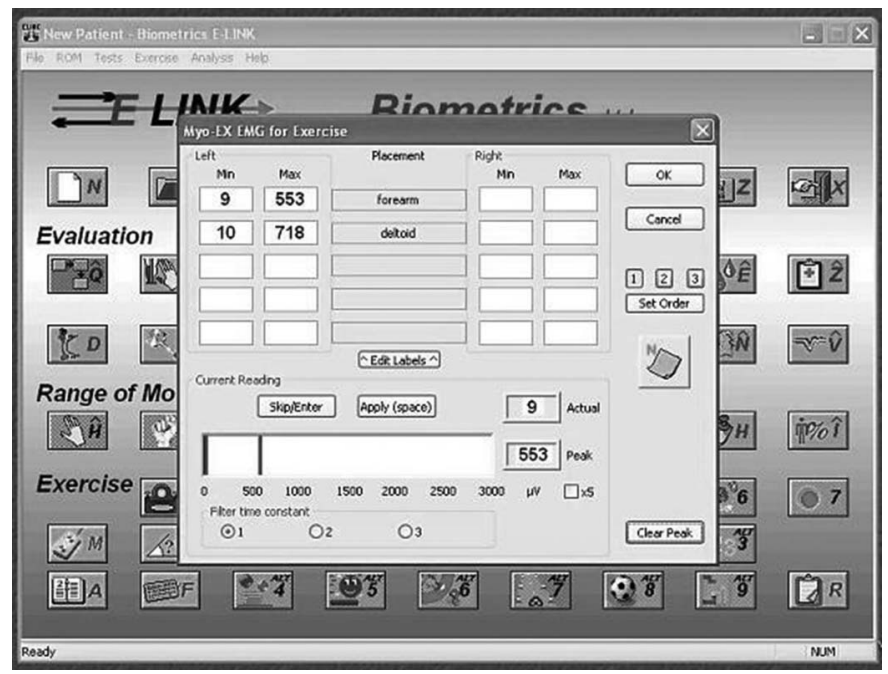

Fig. 2. Biometrics system screen showing the forearm and deltoid EMG in $\mu \mathrm{V}$ in one subject

EMG signal was recorded during a single series of $30 \mathrm{~s}$ vibrations. EMG baseline and maximum value during WBV were recorded in $\mu \mathrm{V}$ (Fig. 2).

\section{Statistical analysis}

Shapiro-Wilk test was used to calculate the sample normality of data. Wilcoxon test was applied to compare the mean values of EMGd (anterior deltoid) and EMGf (forearm muscle). Statistical significance level was assumed if $p<0.05$. IBM-SPSS software version 22 package for Windows (Chicago, IL, USA) was used for all statistical tests.

\section{Results}

A single session of WBV led to a significant increase of EMGd and EMGf activities compared with baseline values $(p<0.01)$ collected under the non-vibration condition (Fig. 3).

EMGd mean value was $745 \mu \mathrm{V}$ during $\mathrm{WBV}$ and $3.2 \mu \mathrm{V}$ during baseline. EMGf mean value during $\mathrm{WBV}$ was $556 \mu \mathrm{V}$, while the baseline was $3.7 \mu \mathrm{V}$ (Fig. 4).

These results showed an increase of over 200 times the EMG signal when comparing WBV with baseline.

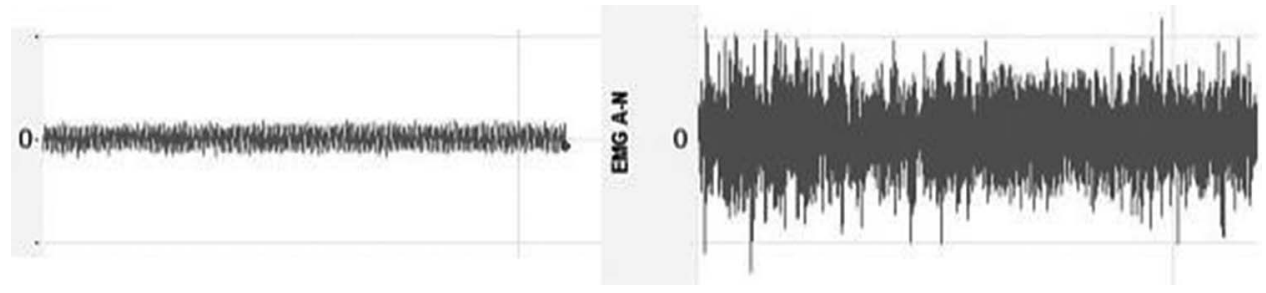

Fig. 3. Difference between the EMG activity baseline (left) and the EMG activity during WBV (right) in one subject 


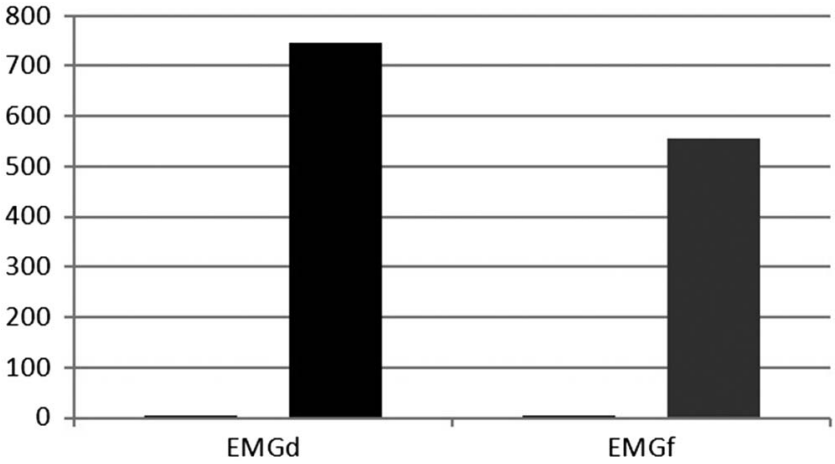

Fig. 4. Graphic of the EMG activities (mean of all subjects). The first counter represents EMGd baseline $(3.2 \mu \mathrm{V})$. The second counter represents EMGd values during WBV that reached a mean of $745 \mu \mathrm{V}$. The third counter represents EMGf value at baseline $(3.7 \mu \mathrm{V})$, while the fourth one shows EMGf during WBV $(556 \mu \mathrm{V})$

\section{Discussion}

Training on vibration plates has become a steadily increasing field of interest in sports science and research. The mechanical vibration stimulus applied to the muscles and tendons during vibration training leads to a neuromuscular response (2). WBV is based, despite a few modifications, on a vertical sinusoidal vibration generating platform working at frequencies between 25 and $40 \mathrm{~Hz}$ with $30 \mathrm{~Hz}$ providing the best results. The platform generates a mechanical stimulus that is transmitted throughout the body where it stimulates sensory receptors such as the muscle spindle (4). As a result, contractions similar to those that take place in the tonic vibration reflex (TVR) through the activation of alpha motor neurons are generated. In the beginning, WBV was directed toward improving the strength velocity performance of elite athletes. Nowadays, however, its use is being more and more generalized and reaching more users (4).

This physiological mechanism has been named "tonic vibration reflex", where muscle spindle reflexes facilitate the activation of Ia motoneurons, leading to muscle contractions. Monosynaptic and polysynaptic pathways have been shown to mediate TVR (8).

Several studies have examined the possibility of using WBV to increase muscle power during arm flexion movement $(1,3)$. Vibrations are starting to be used as an alternative training means for enhancing strength/power characteristics (2).

Results produced by WBV are not free of contradictions. One of the reasons for such contradictions is the different frequencies applied and different training protocols of the studies (4). Inconsistencies in the literature may be due to differences in the amplitude and frequency settings used to induce the WBV stimulus (10). Numerous studies have indeed observed an increase of sEMG recordings of lower limb muscles during WBV compared to no vibration $(3,10,16)$. Unfortunately, inconsistent observations have been reported depending on the vibration frequency, the peak-to-peak displacement of the platform, and the muscle examined $(10,13,16)$.

In fact, previous studies have shown that WBV exercises have shown positive effects in increasing muscle strength and performance (18-21). Muscle spindle Ia reflex has been indicated as the major determinant of this vibration-induced neuromuscular activation leading to $\operatorname{TVR}(2,3,12)$.

WBV has appeared increasingly in scientific journals and is being incorporated into regular training programs aiming to improve physical fitness (5). Several studies have looked at the effects of WBV on muscle performance. However, the results are not clear and are 
sometimes contradictory. The variations in protocols used by different authors may explain the inconsistency of the results presented in the published studies (5).

In sEMG, motion artifacts arise from skin stretch, cable movements, or the relative movement of electrodes. The type and characteristics of electrodes may also influence their occurrence and relevance (7). The sEMG spectrum extends in a wider range between 20 and $500 \mathrm{~Hz}(7)$.

In this context, several studies have investigated the responsiveness of different muscles in different exercises during vibration stimulus using EMG $(1,18)$. It has been reported that a vibration stimulus applied to the arm increased the neuromuscular activity during elbow flexion compared to the same exercise without vibration $(1,18)$.

In this study, a significant increase of EMG activity had been observed on upper limb in individuals with SCI during one single session of WBV. The position assumed over the platform was an easy way to make SCI individuals feel comfortable. The volunteers reported no problems with the posture over the platform or the frequency and said they would like to continue this treatment in the future. Only three of them felt a little discomfort (but not intolerable) about the high frequency of $30 \mathrm{~Hz}$. We believe this little discomfort in a few individuals is due to a personal perception of vibrations as none of them had used WBV before and it could unleash sensations that they were not accustomed to.

The increase of the EMG signals support that the chosen position, over the elbows and forearm, is an effective position for trigging the upper limb muscle spindle.

Our study is in agreement with the previous studies that also found an increase of EMG activity during WBV over upper limb muscles $(8,9,11,15)$ confirming that WBV is an effective tool to increase the muscular activity of upper limb. This study has shown that WBV is also effective in distinct subjects other than healthy, who have been the focus of studies until this point.

In this study, we have tried to avoid situations that had led to the limitations in previous studies. We used the parameters that received the best results on upper limb EMG and it led to positive results.

According to the results, WBV had activated shoulder and forearm muscles. Once these muscles respond positively to WBV, this study opens a new horizon of possibilities to further studies applying WBV to therapeutic and sport uses.

\section{Conclusions}

In conclusion, this study indicates that one single session of WBV significantly improves the EMG activity of the upper limb muscles in individuals with SCI. These results support WBV as an alternative tool to be used in SCI rehabilitation and training and its effectiveness in subjects other than healthy. The position assumed during the WBV was effective and comfortable for individuals with SCI. The chosen parameters, based on preview studies, had avoided limitations and contradictions.

Shoulder pain and joint issues can be treated and avoided when its muscles are safe and strong. This study shows that WBV activates these muscles positively and it can be applied in further therapeutic and sports researches.

Further studies are needed to understand the long-term effects of WBV in the same individuals with SCI. 


\section{REFERENCES}

1. Bosco C, Cardinale M, Tsarpela O: Influence of vibration on mechanical power and electromyogram activity in human arm flexor muscles. Eur. J. Appl. Physiol. Occup. Physiol. 79, 306-311 (1999)

2. Cardinale M, Lim J: Electromyography activity of vastus lateralis muscle during whole-body vibrations of different frequencies. J. Strength Cond. Res. 17(3), 621-624 (2003)

3. Cormie P, Deane RS, Triplett NT, McBride JM: Acute effects of whole-body vibration on muscle activity, strength, and power. J. Strength Cond. Res. 20(2), 257-261 (2006)

4. Da Silva ME, Nuñez VM, Vaamonde D, Fernandez JM, Poblador MS, Garcia-Manso JM, Lancho JL: Effects of different frequencies of whole body vibration on muscular performance. Biol. Sport 23(3), 267-282 (2006)

5. Da Silva-Grigoletto ME, De Hoyo M, Sanudo B, Corrales L, Garcia-Manso JM: Determining the optimal wholebody vibration dose-response relationship for muscle performance. J. Strength Cond. Res. 25(12), 3326-3333 (2011)

6. Fratini A, Bifulco P, Romano M, Clemente F, Cesarelli M: Simulation of surface EMG for the analysis of muscle activity during whole body vibratory stimulation. Comput. Methods Programs Biomed. 113, 314-322 (2014)

7. Fratini A, La Gatta A, Bifulco P, Romano M, Cesarelli M: Muscle motion and EMG activity in vibration treatment. Med. Eng. Phys. 31, 1166-1172 (2009)

8. Gyulai G, Rácz L, Di Giminiani R, Tihanyi J: Effect of whole body vibration applied on upper extremity muscles. Acta Physiol. Hung. 100(1), 37-47 (2013)

9. Hang J, Verscheure S, Osternig L: A comparison of whole-body vibration and resistance training on total work in the rotator cuff. J. Athl. Train. 44(5), 469-474 (2009)

10. Hazell TJ, Jakobi JM, Kenno KA: The effects of whole-body vibration on upper and lower body EMG during static and dynamic contractions. Appl. Physiol. Nutr. Metab. 32, 1156-1163 (2007)

11. Hong J, Velez MT, Moland AM, Sullivan JA: Acute effects of whole body vibration on shoulder muscular strength and joint position sense. J. Hum. Kinet. 25, 17-25 (2010)

12. Lienhard K, Cabasson A, Meste O, Colson SS: Determination of the optimal parameters maximizing muscle activity of the lower limbs during vertical synchronous whole-body vibration. Eur. J. Appl. Physiol. 114, 1493-1501 (2014)

13. Lienhard K, Cabasson A, Meste O, Colson SS: sEMG during whole-body vibration contains motion artifacts and reflex activity. J. Sports Sci. Med. 14, 54-61 (2015)

14. Marín PJ, Hazell TJ: Effects of whole-body vibration with an unstable surface on muscle activation. J. Musculoskelet. Neuronal Interact. 14(2), 213-219 (2014)

15. Marín PJ, Herrero AJ, Milton JG, Hazell TJ, Garcia-Lopes D: Whole-body vibration applied during upper body exercise improves performance. J. Strength Cond. Res. 27(7), 1807-1812 (2013)

16. Marín PJ, Santos-Lozano A, Santin-Medeiros F, Vicente-Rodriguez G, Casajús JA, Hazell TJ, Garatachea N: Whole-body vibration increases upper and lower body muscle activity in older adults: potential use of vibration accessories. J. Electromyogr. Kinesiol. 22, 456-462 (2012)

17. Mulroy SJ, Farrokhi S, Newsam CJ, Perry J: Effects of spinal cord injury level on the activity of shoulder muscles during wheelchair propulsion: an electromyographic study. Arch. Phys. Med. Rehabil. 85, 925-934 (2004)

18. Perchthaler D, Horstmann T, Grau S: Variations in neuromuscular activity of thigh muscles during whole-body vibration in consideration of different biomechanical variables. J. Sports Sci. Med. 12, 439-446 (2013)

19. Reyes GFC, Dickin DC, Crusat NJK, Dolny DG: Whole-body vibration effects on the muscle activity of upper and lower body muscles during the baseball swing in recreational baseball hitters. Sports Biomech. 10(4), 280-293 (2011)

20. Ritzmann R, Kramer A, Gruber M, Gollhofer A, Taube W: EMG activity during whole body vibration: motion artifacts or stretch reflexes? Eur. J. Appl. Physiol. 110, 143-151 (2010)

21. Stewart JA, Cochrane DJ, Morton RH: Differential effects of whole body vibration durations on knee extensor strength. J. Sci. Med. Sport 12, 50-53 (2009) 